\title{
Thermo-Optic Effects in an Electro-Optic Modulation System
}

\author{
M._Aillerie ${ }^{1}$, N. Théofanous ${ }^{2}$, H. L. Saadon ${ }^{2}$ \\ ${ }^{1}$ LMOPS, UMR CNRS 7132, Université Paul Verlaine - Metz and Supélec, 2, rue E. Belin, \\ 57070 Metz, France \\ ${ }^{2}$ Department of Informatics and Telecommunications, Optoelectronics Group, University of \\ Athens, Panepistimiopolis, Ilisia, GR-15784 Athens, Greece \\ aillerie@,metz.supelec.fr
}

\section{Introduction}

Thermo-optic (TO) and relevant temperature effects in intensity-modulation (IM) electro-optic (EO) systems may strongly affect the EO response of a crystal [1-3]. In the entire EO- modulation system, these effects can be examined via their influence on the position and thermal stability of the operating point over the system's characteristic (transfer) curve [3].

In this framework, our interest has been focused to the thermo-optic behaviour of an EO modulator crystal as it is expressed by the influence of temperature T on crystal's optical or electro-optical parameters such as the refractive indices, $\mathrm{n}_{0}$ and $\mathrm{n}_{\mathrm{e}}$, the effective EO coefficient $r$, the spontaneous birefringence $\Delta \mathrm{n}(0)$, the half-wave voltage $\mathrm{V}_{\pi}$, the optical phase retardation $\Gamma$, and the intensity-modulation (IM) depth $\mathrm{m}$ of the entire EO-modulation system. After a brief presentation of typical configurations of IM systems, we define the principal thermo-optic and temperature coefficients and we derive useful general equations connecting these parameters in the entire system. Then we present investigations of these equations as regards the above parameters along with calculations of the uncertaintities in the determination of temperature coefficients for the effective EO coefficient $r$, the half-wave voltage $\mathrm{V}_{\pi}$, and the IM depth $\mathrm{m}$. It is of note that in this publication, the theoretical analysis of the thermo-optic effects in an electro-optic modulation system is only presented. In the experimental field, a determination of various TO coefficients of $\mathrm{LiTaO}_{3}$ made by the authors, in the case of a transverse EO configuration, has been already published elsewhere [3].

\section{Typical configurations of the system}

In general, for Intensity Modulation (IM) systems, the transfer function for the transmitted light in tensity I can be put in the form $\mathrm{I}=\mathrm{f}(\varphi)$, where $\varphi$ is a quantity we have called the opto-electrical bias of the system and is defined by [3]

$$
\varphi=\Gamma-2 \beta \text {. }
$$

In Fig. 1 we depict the structure of a typical IM system based on a Sénarmont configuration set-up. For such systems the transfer function becomes $[3,6]$

$$
\mathrm{T}=\frac{\mathrm{I}_{0} \mathrm{~T}_{0}}{2}(1-\sin \varphi)
$$

where $I_{o}$ denotes the intensity of the (unpolarized) light incident to the input polarizer and $T_{o}$ is the transmission factor of the system.

In what follows, the crystal sample is assumed linearly electro-optic and, therefore, when an electric field $\mathrm{E}=\mathrm{V} / \mathrm{d}$ (where $\mathrm{V}$ is the applied voltage and $\mathrm{d}$ the electrode spacing) is applied to the crystal, a field-induced birefringence [7,8]

$$
\Delta \mathrm{n}_{\mathrm{E}}=\frac{1}{2} \mathrm{n}^{3} \mathrm{rE}=\frac{\mathrm{n}^{3} \mathrm{r}}{2 \mathrm{~d}} \mathrm{~V},
$$

is superimposed on the natural birefring ence $\Delta n(0)$. In this equation, $r$ represents the effective 
EO coefficient and $n$ the active refractive index of the sample. Applying Eqs.3 to the wellknown formula

$$
\Gamma=\left(\frac{2 \pi \mathrm{L}}{\lambda_{0}}\right) \Delta \mathrm{n},
$$

where $\mathrm{L}$ is the length of the crystal (along the light beam) and $\lambda_{0}$ the wavelength of the beam in a vacuum, one obtains for the field-induced optical phase retardation the expression

$$
\Gamma_{\mathrm{E}}=\left(\frac{\pi}{\lambda_{0}}\right) \operatorname{Ln}^{3} \mathrm{rE}=\pi\left(\frac{\mathrm{V}}{\mathrm{V}_{\pi}}\right),
$$

where

$$
\mathrm{V}_{\pi}=\frac{\lambda_{0}}{\mathrm{n}^{3} \mathrm{r}}\left(\frac{\mathrm{d}}{\mathrm{L}}\right)
$$

is the half-wave voltage of the crystal sample for the configuration under consideration.

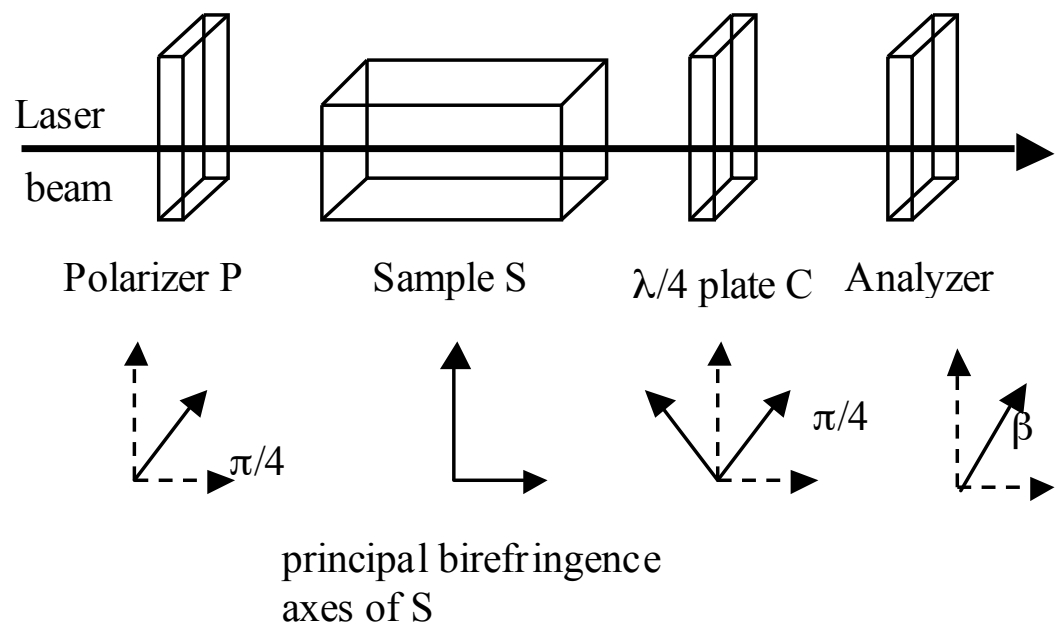

Figure 1 : The typical Intensity Modulation system based on a Sénarmont- type arrangement

Hence, in the case of a transverse EO modulator, the half-wave voltage of the sample will be proportional to the dimension ratio $\mathrm{d} / \mathrm{L}$. Accordingly, in the transverse EO-modulation system under consideration, the half-wave voltage of the sample could be considerably reduced if $d$ is taken significantly smaller than $L$ and, moreover, it would be more or less affected by the thermal expansion effects in the crystal. By contrast, for the longitudinal configuration we have $\mathrm{L}=\mathrm{d}$ and therefore the half-wave voltage of the crystal is independent on its dimensions, and their thermal expansions, and depends only on the thermo-optic effects modifying the refractive index and the EO coefficient of the crystal.

When a system such as the one shown in Fig. 1 is used for either a transverse or a longitudinal IM electro-optic modulation, the I- $\varphi$ curve representing the transfer function according to Eq. 2 can be taken as the characteristic curve of the system (Fig. 2). 


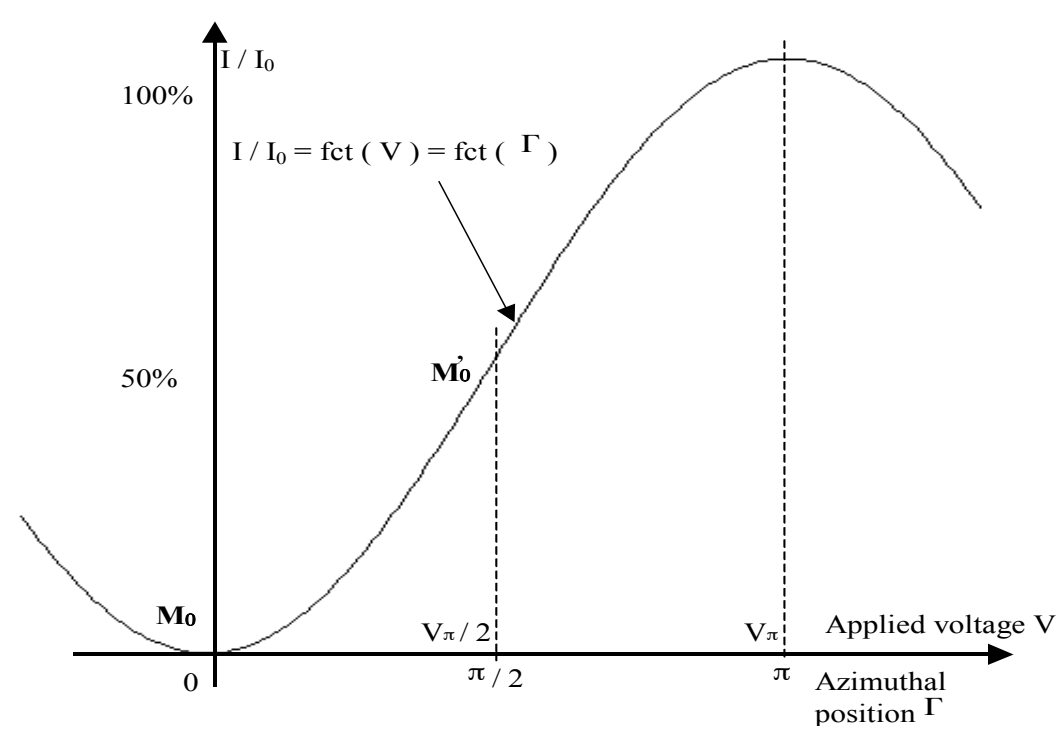

Figure 2 : Characteristic curve of the $\mathrm{IM}$ system, representing the transmission $\mathrm{T}=\mathrm{I} / \mathrm{I}_{\mathrm{o}}$ as a function of the applied voltage $\mathrm{V}$ and as a function the of total phase retardation $\Gamma$.

As it can be seen from Eqs. 1-5, the position of the quiescent point on this characteristic curve is controlled by the opto-electrical bias $\varphi$ and can be established by adjusting either the azimuthal angle $\beta$ of the analyzer (optical bias) or/and the applied electric dc voltage $V=V_{d c}$ (electrical bias). The later which commands the static optical retardation $\bar{\Gamma}=\Gamma^{(0)}+\Gamma_{\mathrm{dc}}$, in which $\Gamma^{(0)}$ is the natural phase retardation and $\Gamma_{\mathrm{dc}}=\pi\left(\mathrm{V}_{\mathrm{dc}} / \mathrm{V}_{\pi}\right)$ is the dc-bias phase retardation. By virtue of Eq. (1), the to tal op to-electrical bias will be giv en by the expression

$$
\bar{\varphi}=\bar{\Gamma}-2 \beta=\Gamma^{(0)}+\pi\left(\mathrm{V}_{\mathrm{dc}} / \mathrm{V}_{\pi}\right)-2 \beta \text {. }
$$

\section{Relationships between TO parameters of EO interest}

In an EO-modulation system, various thermo-optic (TO) parameters exist which refer to the temperature dependence of useful quantities involved in the operation of the EO system. Clearly, as $\Gamma$ depends on various EO and TO parameters, the thermal stability of the optoelectrical bias $\bar{\varphi}=\bar{\Gamma}-2 \beta$ will depend on the values of these parameters. To describe the thermal behavior of a temperature dependent quantity $u$ [3], we have adopted the definitions $\mathrm{p}=\mathrm{du} / \mathrm{dT}$ for the thermo-optic (TO) coefficient and

$$
q=\left(\frac{1}{u}\right)\left(\frac{d u}{d T}\right),
$$

for the corresponding temperature coefficient. Following the above, we have defined the parameters labeled $\rho, \delta, \mu_{\Delta}$, and $\xi_{\mathrm{m}}$ as the temperature coefficients for the effective EO coefficient $r$, the half-wave voltage $V_{\pi}$, the spontaneous birefringence $\Delta n^{(0)}$, and the IM depth $\mathrm{m}$, respectively. E.g., we have defined the following coefficients

$$
\delta=\frac{1}{\mathrm{~V}_{\pi}}\left(\frac{\mathrm{dV}_{\pi}}{\mathrm{dT}}\right), \quad \mu_{\Delta}=\frac{1}{\Delta \mathrm{n}^{(0)}}\left(\frac{\mathrm{d} \Delta \mathrm{n}^{(0)}}{\mathrm{dT}}\right),
$$

where $\mathrm{dV}_{\pi} / \mathrm{dT}$ and $\mathrm{d} \Delta \mathrm{n}^{(0)} / \mathrm{dT}$ are the thermo-optic coefficients for $\mathrm{V}_{\pi}$ and $\Delta \mathrm{n}^{(0)}$, respectively. Also, the temperature coefficients of the refractive indices $\mathrm{n}_{1}=\mathrm{n}_{0}$ and $\mathrm{n}_{2}=\mathrm{n}_{\mathrm{e}}$, respectively, have been defined by the expressions 


$$
\beta_{1}=\beta_{0}=\frac{1}{\mathrm{n}_{0}}\left(\frac{\mathrm{dn}_{0}}{\mathrm{dT}}\right), \quad \beta_{2}=\beta_{\mathrm{e}}=\frac{1}{\mathrm{n}_{\mathrm{e}}}\left(\frac{\mathrm{dn}_{\mathrm{e}}}{\mathrm{dT}}\right),
$$

where $\mathrm{dn}_{0} / \mathrm{dT}$ and $\mathrm{dn}_{\mathrm{e}} / \mathrm{dT}$ represent the corresponding thermo-optic coefficients. Lastly, $\overline{\mathrm{n}}=(1 / 2)\left(\mathrm{n}_{1}+\mathrm{n}_{2}\right)$ is the mean refractive index and $\kappa$ represents the thermo-optic coefficient $\mathrm{d} \bar{\Gamma} / \mathrm{dT}$ of the static phase retardation $\bar{\Gamma}$. As to the temperature coefficients $\alpha_{\mathrm{L}}$ and $\alpha_{\mathrm{d}}$ for the crystal length $\mathrm{L}$ and the crystal thickness $\mathrm{d}$, respectiv ely, they are defined by

$$
\alpha_{\mathrm{L}}=\frac{1}{\mathrm{~L}}\left(\frac{\mathrm{dL}}{\mathrm{dT}}\right), \quad \alpha_{\mathrm{d}}=\frac{1}{\mathrm{~d}}\left(\frac{\mathrm{dd}}{\mathrm{dT}}\right),
$$

where $\mathrm{dL} / \mathrm{dT}$ and $\mathrm{dd} / \mathrm{dT}$ are the thermal expansion coefficients of the crystal along the directions $\mathrm{L}$ and $\mathrm{d}$, respectively.

In the case of a Sénarmont-type transverse EO-modulation system, it has been proven that the following set of equations is valid for the EO and TO parameters for any operating point of the system [3]:

$$
\begin{aligned}
& \mathrm{n} \mathrm{n}_{12}\left(\beta_{2}-\beta_{1}\right)=\overline{\mathrm{n}} \Delta \mathrm{n}^{(0)}\left(\mu_{\Delta}-\bar{\mu}\right) \\
& \kappa=\frac{\mathrm{d} \bar{\Gamma}}{\mathrm{dT}}=\Gamma^{(0)}\left(\mu_{\Delta}+\alpha_{\mathrm{L}}\right)-\pi \delta\left(\frac{\mathrm{V}_{\mathrm{dc}}}{\mathrm{V}_{\pi}}\right) \\
& \rho=\frac{1}{\mathrm{r}}\left(\frac{\mathrm{dr}}{\mathrm{dT}}\right)=-\left(\delta+3 \beta_{\mathrm{e}}\right)+\left(\alpha_{\mathrm{d}}-\alpha_{\mathrm{L}}\right) \\
& \xi_{\mathrm{m}}=\frac{1}{\mathrm{~m}}\left(\frac{\mathrm{dm}}{\mathrm{dT}}\right)=\left(\rho+3 \beta_{\mathrm{e}}\right)-\left(\alpha_{\mathrm{d}}-\alpha_{\mathrm{L}}\right)
\end{aligned}
$$

Similar calculations made in the case of a Senarmont-type longitudinal EO-modulation system have been shown that Eqs.14a and $14 \mathrm{~b}$, which refer to the difference $\left(\beta_{2}-\beta_{1}\right)$ and to the thermo-optic coefficient $\kappa$ of $\bar{\Gamma}$, respectively, are the same in both the transverse and the longitudinal EO system. By contrast, Eqs. 14c and 14d, which refer to the temperature coefficients $\rho$ and $\xi_{m}$, respectively, differ in these two opto-geometric configurations by the thermal expansion term $\left(\alpha_{\mathrm{d}}-\alpha_{\mathrm{L}}\right)$, which is absent in the longitudinal EO-modulation system. This fact may be advantageous for the longitudinal EO-modulation system, particularly if the thermal expansion contribution is high in the corresponding transverse EO-modulation system.

\section{Investigation and discussion}

We first deal with the difference $\beta_{2}-\beta_{1}$ of temperature coefficients for the two birefringence refractive indices and the thermo-optic coefficient $\kappa$ of the static phase retardation, which are ruled by Eqs. $14 \mathrm{a}$ and $14 \mathrm{~b}$. It is again to be emphasized that, irrespective of the chosen EO-modulation configuration (transverse or longitudinal) these parameters are the same.

Besides, from Eq. 14a we observe that, if $\Delta \mathrm{n}^{(0)}=0$, which corresponds to an initially isotropic crystal or a propagation along an optical ax is, we shall have $\beta_{1}=\beta_{2}$. Consequently, when the two refractive indices $\mathrm{n}_{1}$ and $\mathrm{n}_{2}$ are equal, they will have also equal temperature coefficients. On the other hand, from Eq. $14 \mathrm{~b}$ we deduce that the thermo-optic coefficient $\kappa$ will be zero when $\Gamma^{(0)}\left[\mu_{\Delta}+\alpha_{\mathrm{L}}\right]=\pi \delta\left[\mathrm{V}_{\mathrm{dc}} / \mathrm{V}_{\pi}\right]$. Consequently, under this condition, the static phase retardation $\bar{\Gamma}$ will be thermally insensitive. 
Moreover, we consider the temperature coefficient $\rho$ of the crystal's EO coefficient $r$ and the temperature coefficient $\xi_{\mathrm{m}}$ of the modulation depth $\mathrm{m}$, which are ruled by Eqs. $14 \mathrm{c}$, and $14 \mathrm{~d}$. For these parameters, one can discriminate three cases as follows:

1) $\alpha_{d}=\alpha_{L}$. In this case, the temperature coefficients $\rho$ and $\xi_{m}$ will be the same for both transverse and longitudinal EO-modu lation configurations.

2) $\alpha_{d}>\alpha_{L}$. From Eqs $14 \mathrm{c}$ and $14 \mathrm{~d}$ it is deduced that $\rho_{\text {trans }}>\rho_{\text {long }}$ and $\xi_{\mathrm{m}, \text { trans }}>\xi_{\mathrm{m}, \mathrm{long}}$. This means that, as regards the thermal stability of $\mathrm{r}$ and $\mathrm{m}$, the transverse EO-modulation system will then be worse than its homologous longitudinal EO-modulation system.

3) $\alpha_{d}<\alpha_{L}$. It is the opposite of case 2 . Thus, it is deduced that $\rho_{\text {trans }}<\rho_{\text {long }}$ and $\xi_{\text {m,trans }}<\xi_{\text {m,long }}$, which means that the transverse EO-modulation system will then be better than its homologous longitudinal EO-modulation system.

\section{Summary and conclusion}

The first part of the present work has been dedicated to in troducing and analyzing the concept of opto-electrical bias $\varphi$ in an EO-modulation system. Next, we have dealt with corresponding questions of thermal instability, as it is expressed by various thermo-optic (TO) or temperature-d ependence parameters.

It has been thus proven that the choice of a transverse or a longitudinal configuration in the system may more or less affect the above parameters and modify the thermal stability of the EO crystal and the entire system as well. Also, a comparison between the transverse and longitudinal EO-modulation systems, as regards the thermo-optic behavior of the EO crystal and the entire set-up, has been effected and discussed.

\section{Acknowledgements}

The project of this work is co-funded by the European Social Fund and National Resources - EPEAEK II PYTHAGORAS. The authors wish to acknowledge the corresponding organizations for this support.

\section{References}

[1] Rauber A: In Current Topics in Material Sciences. North Holland, Amsterdam; 1978.

[2] Chen F S: Modulators for Optical Commun ications. Proc IEEE. 1970;58: 1440-1457.

[3] Saadon H L, Theofanous N, Aillerie M, Fontana M D: Thermo-Optic Effects in ElectroOptic Crystals Used in an Intensity-Modulation System.-Application in $\mathrm{LiTaO}_{3}$. Appl. Phys. B. 2006;83: 609-617.

[4] Kaminow I P, Turner E H: Electrooptic Light Modulators. Proc. IEEE. 1966;54: 13741390.

[5] Aillerie M, Fontana M D, Abdi F, Carabatos C, Theofanous N, Alexakis G: Influence of the Temperature-Dependent Spontaneous Birefringence in the Electro-Optic Measurements of $\mathrm{LiNbO}_{3}$. J. Appl. Phys. 1989; 65: 2406-2408.

[6] Theochar is P S, Gdoutos E E: Matrix Theory of Photoelasticity. Springer; Berlin; 1979.

[7] Yariv A, Yeh P: Optical Waves in Crystals. New York: Wiley; 1984.

[8] Saadon H L, Theofanous N, Aillerie M, Abarkan M, Salvestrini J P, Fontana M D: The Electro-Optic $\mathrm{r}_{22}$ Coefficients and Acoustic Contributions in a $\mathrm{LiTaO}_{3}$ Crystal. J. Opt. A: Appl. Opt. 2006;8: 677-682.

[9] Theofanous N, Aillerie M, Fontana M D, Alexakis G: Frequency Doubling Electro-Optic Modulation System for Pockels Effect Measurements: Application in $\mathrm{LiNbO}_{3}$. Rev. Sci. Instrum. 1997;68: 2138-2143.

[10] Aillerie M, Theofanous N, Fontana M D: Measurement of the Electro-Optic Coefficients: Description and Comparison of the Experimental Techniques. Appl. Phys. B. 2000;70: 317334. 